\title{
The biology of HIV infection
}

\author{
SALLY COWLEY \\ Open University, Department of Biological Sciences, Walton Hall, \\ Milton Keynes MK7 6AA, UK
}

\begin{abstract}
Summary This article reviews the cell and molecular biology of human immunodeficiency virus (HIV), emphasizing the features that lead to opportunistic infection by organisms such as mycobacteria. Mycobacteria, especially $M$. avium complex and M. tuberculosis infections, are closely associated with HIV disease. HIV is a very small retrovirus and its high mutation rate leads to extremely variable viral populations, both within and between individuals. It is coated with glycoprotein 120 (gp120), which it uses to bind to and infect a range of CD4+ leukocytes, depending on the co-receptor specificity. T cell-tropic HIV strains tend to use the CXCR-4 chemokine receptor, while macrophage-tropic strains tend to use the CCR-5 chemokine receptor. Immunosuppression is induced in a number of ways. As well as frank depletion of virus-infected $\mathrm{T}$ cells, antigen-specific $\mathrm{T}$ cell clones can be selectively deleted by mechanisms such as defective antigen presentation by HIV-infected macrophages (activation-induced cell death). Changes in cytokine production in HIV infection are also proposed. All this leads to falling T cell counts, B cell dysregulation and macrophage dysfunction. Opportunistic infections exploit this immunosuppressed environment. Certain infections are prevalent, reflecting factors such as environmental exposure to pathogens, poor mucosal defences and subcellular interactions between HIV and, e.g. viral or mycobacterial infections. Opportunistic infection exacerbates immune destruction by HIV, producing a vicious cycle that is ultimately fatal.
\end{abstract}
Abbreviations
HIV
AIDS
human immunodeficiency virus
MAC acquired immunodeficiency syndrome
CD4
Mycobacterium avium complex
HLA
cluster of differentiation antigen 4
RNA
human leukocyte antigen
ribonucleic acid

Correspondence to: S. Cowley, 106 High Street, Long Crendon, Bucks HP18 9AN, UK (e-mail sally.cowley@virginnet.co.uk) 


$\begin{array}{ll}\text { DNA } & \text { deoxyribonucleic acid } \\ \text { TCR } & \text { T cell receptor } \\ \text { TNF- } \alpha & \text { tumour necrosis factor } \alpha \\ \text { gp120 } & \text { HIV glycoprotein } 120 \\ \text { APC } & \text { antigen presenting cell } \\ \text { MHC } & \text { major histocompatibility antigen } \\ \text { M tropic } & \text { macrophage tropic HIV strains } \\ \text { T tropic } & \text { T cell tropic HIV strains } \\ \text { CSF } & \text { cerebrospinal fluid } \\ \text { RANTES } & \text { regulated on activation, normal T expressed and secreted chemokines } \\ \text { MIP-1 } \alpha & \text { macrophage inflammatory protein } 1 \alpha \\ \text { CXC } & \text { chemokines containing a cysteine-any amino acid-cysteine motif } \\ \text { CXCR } & \text { CXC chemokine receptor } \\ \text { X4 tropic } & \text { HIV strains that bind to CXCR-4 } \\ \text { CC } & \text { chemokines containing two adjacent cysteine molecules } \\ \text { CCR } & \text { CC chemokine receptor } \\ \text { R5 tropic } & \text { HIV strains that bind to CCR-5 } \\ \text { R5X4 } & \text { HIV strains that bind to both CXCR-4 and CCR-5 } \\ \text { NSI } & \text { non-syncytium inducing HIV strains } \\ \text { AICD } & \text { activation-induced cell death } \\ \text { IL-10 } & \text { interleukin-10 } \\ \text { Th-1 } & \text { T helper subset } 1 \\ \text { IgG } & \text { immunoglobulin G } \\ \text { LAM } & \text { mycobacterial lipoarabinomannan } \\ \text { H37Ra } & \text { avirulent strain of } M \text {. } t b \\ \text { H37Rv } & \text { virulent strain of } M \text {. t } b \\ & \end{array}$

\section{Introduction}

There are more than 30 million people living with human immunodeficiency virus (HIV) worldwide. ${ }^{1}$ As the immune system is progressively compromised and HIV infection develops into clinical acquired immunodeficiency syndrome (AIDS), many opportunistic infections are encountered, including some important mycobacteria.

Mycobacterium avium complex (MAC) is ubiquitous in the environment and commonly causes serious opportunistic disseminated infection in AIDS patients who have low circulating CD4 T cell counts $(<50$ cells $/ \mathrm{ml}){ }^{2}$

Mycobacterium tuberculosis (M.tb) is also a frequent cause of morbidity and mortality in HIV-infected patients. With a third of the world latently infected with TB, especially in nonindustrialized countries, much of the HIV-related TB is due to reactivation of dormant bacilli, often in the earlier stages of HIV disease (CD4 counts of $>300$ cells $/ \mathrm{ml}$ ). Primary infection is also a problem, especially in patients with low CD4 cell counts, and, alarmingly, nosocomial outbreaks (including with multidrug resistant strains) have occurred in healthcare settings. ${ }^{3,4}$ There is a less well documented association between leprosy and HIV, but the two diseases coexist geographically and co-infection does occur. ${ }^{5-8}$

It is clear then that health professionals and researchers involved with mycobacteria need a working understanding of HIV disease. This article in the series aims to review and give an 
update on the biology of HIV, highlighting the cellular events behind the clinical association between HIV and opportunistic infections such as mycobacteria.

\section{The HIV virus - structure and replication}

The structure of the HIV viruses helps us understand why they mutate so much and how they infect and multiply in particular cell types.

HIV-1 and HIV-2 are retroviruses, and are impressively small, simple viruses. The genome is a mere 9.8 kilobases, coding for just a dozen proteins. The core and matrix of viral proteins are surrounded by a lipid envelope, which is derived from the host cell as the virus particle buds off the cell. It can contain host cellular proteins such as HLA and these help to mask the virus from the immune system. The envelope is also studded with viral envelope glycoproteins, which are crucial in infectivity and immunogenicity, as we shall see below. HIV-2 is about 55\% sequence divergent from HIV-1 and is serologically distinct, mostly due to differences in the envelope glycoprotein. ${ }^{9}$

HIV uses the envelope protein gp120 to bind to cellular receptors (CD4 and a coreceptor) and fuse with target cells, particularly $\mathrm{T}$ cells and macrophages (about which much more later). Once inside the host cell, the viral genome and reverse transcriptase are unpacked and the RNA is reverse transcribed into DNA (see Figure 1). Reverse transcriptase is relatively error-prone, so viral variants are continually produced. This variability underlies many of the features of HIV infection (see next section). The transcribed DNA is then transported to the nucleus and randomly integrated into the host genome, and new viral copies are made by hijacking the host's cellular machinery (see Figure 1). This process requires the cell to be activated [e.g. via the $\mathrm{T}$ cell receptor (TCR) ${ }^{10}$ or via the cytokine tumour necrosis factor- $\alpha$ (TNF- $\alpha)],{ }^{9}$ so we can immediately see that activation of the immune response stimulates virus production. This leaves the host in an impossible situation; in attempting to eliminate the virus (or other opportunistic infections), it can actually trigger further virus production.

\section{HIV diversity}

HIV has a very high mutation rate, giving rise to hugely variable viral populations, both within an individual and between individuals.

Variation is most prevalent and important in the major immunogenic envelope protein gp120, where there are several hypervariable regions. Within an individual, the virus can change over time and in different locations within the body. Between individuals, strains can vary by around $10 \%$ of their total sequence and by as much as $40 \%$ of amino acid sequences in gp120. This has led to the classification of global HIV-1 strains into 11 subtypes (A-J and O), also called clades. ${ }^{9}$

As well as making life difficult for epidemiologists, there are at least two major biological implications of gp120 diversity. Firstly, the constantly changing viral antigens means the immune system is always a step behind and is less able to mount an effective response. On a larger scale, this also means the medical community is struggling to find an effective vaccine. Secondly, because gp120 is used to bind to host cells, infectivity of the virus changes between strains and over time within an individual, as described below. 


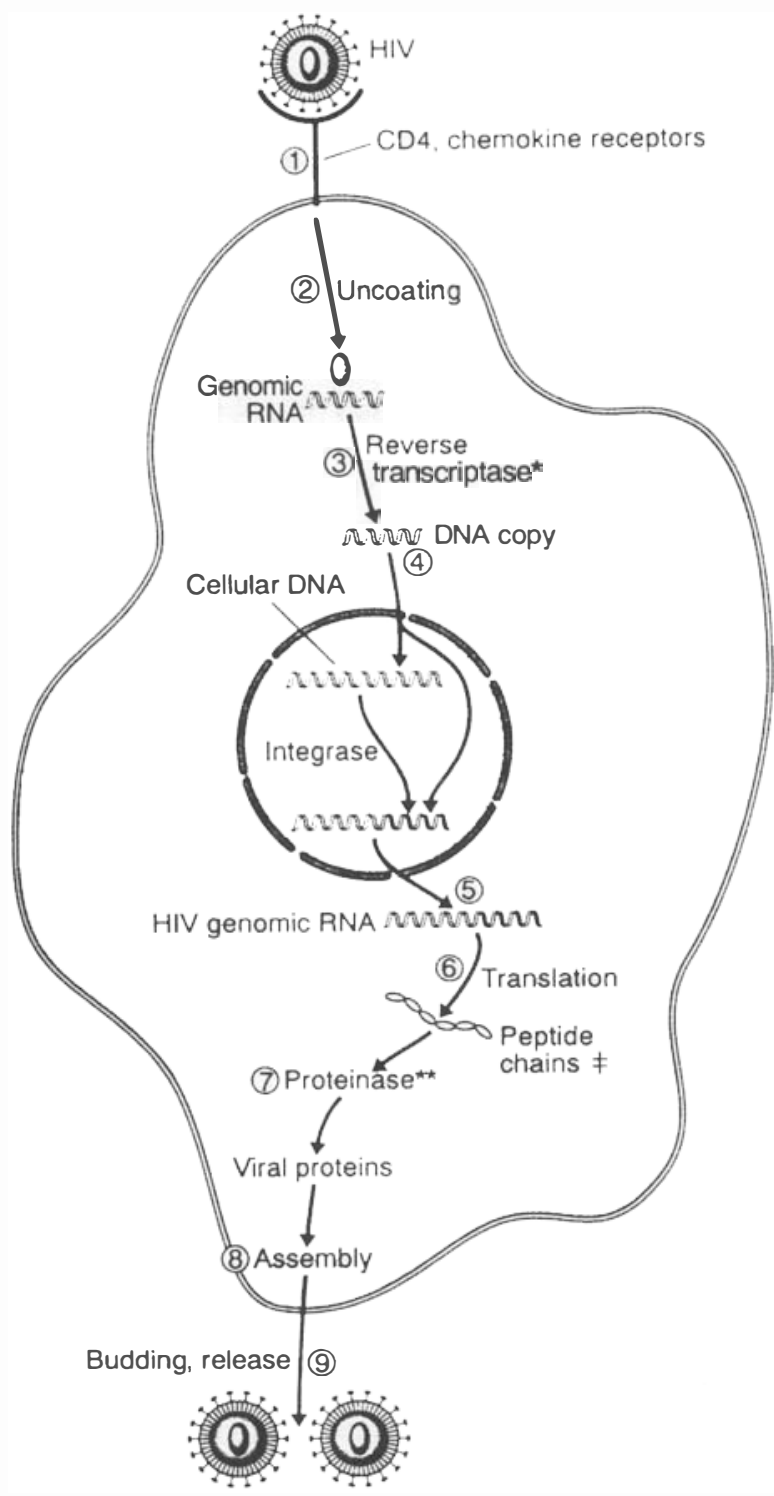

Figure 1. Schematic representation of HIV entry, disassembly, replication and release from susceptible cell (lymphocyte or macrophage). (1) HIV binds to cell surface via receptors (CD4 molecule and coreceptors). (2) Viral uncoating in cytoplasm. (3) RNA viral genome transcribed to DNA copy-reverse transcriptase (RT) enzyme.* (4) DNA copy integrates into host cell genome in cell nucleus via integrase enzyme. (5) Following cell activation viral DNA is translated to RNA copies in cytoplasm. (6) Viral peptide chains translated from cytoplasmic viral RNA. (7) HIV proteinase cleaves functional viral proteins from polypeptides.** (8) Virion assembly. (9) Viral release from cell surface - cell lysis. $* \mathrm{RT}$ inhibitors act here. $* *$ Proteinase inhibitors act here. (Reproduced with permission from Davidson's Principles and Practice of Medicine, 18th edition, Harcourt Brace, 1999.) 


\section{Receptors for HIV}

We now know that CD4 is the major cellular receptor for HIV and that there is also a family of minor coreceptors, which determine the cell types that different HIV strains infect.

It was quite early in HIV research, in 1984, that CD4 was shown to be the major cellular receptor for the virus, binding directly to gp120. ${ }^{11,12} \mathrm{CD} 4$ is present at high levels on most $\mathrm{T}$ cells and also at lower levels on many antigen-presenting cells (APCs), such as macrophages. It associates with the $\mathrm{T}$ cell receptor complex during antigen presentation, binding with MHC class II proteins on the antigen presenting cell and acting as a co-stimulatory molecule.

However, the cellular distribution of CD4 went most, but, crucially, not all the way, to explaining the cellular range of susceptibility to the HIV virus. It was found that some viral isolates were tropic for $\mathrm{T}$ cells ( $\mathrm{T}$-tropic), especially those that had been passaged in $\mathrm{T}$ cell lines in the laboratory). Others were tropic for macrophages (M-tropic). Isolates from patients in early stages of the disease tend to be M-tropic, T-tropic strains appearing in more advanced disease. M-tropic strains are also more able to infect neural cells - patients succumbing to AIDS-related dementia tend to have M-tropic strains of the virus in their brain and CSF. ${ }^{9}$

Then in 1995, Cocchi et al. ${ }^{13}$ showed that infection of cells by M-tropic isolates was inhibited by a group of chemokines known as RANTES (regulated on activation, normal T expressed and secreted), MIP- $1 \alpha$ (macrophage inflammatory protein $1 \alpha$ ) and MIP- $1 \tilde{\beta}$ This prompted a publishing frenzy in 1996 when the second receptor was shown, by molecularly engineered expression, to be various members of a chemokine receptor family. ${ }^{14-17}$ It is the viral gp120 that also binds to these chemokine receptors. ${ }^{18,19}$

At least 10 different chemokine/HIV receptors have now been identified, but the names, classification and specificity can appear confusing. Put simply, there are two groups. CXC chemokines (containing cysteine-any amino acid-cysteine) mostly attract neutrophils and the receptors are designated CXCR. Laboratory-adapted HIV strains, grown for many passages in $\mathrm{T}$ cell lines and which can only infect $\mathrm{T}$ cells (and are often able to induce syncytium formation in host cells), use only CXCR-4 as the coreceptor, so these strains are also referred to as $\mathrm{X} 4$ tropic. Conversely, $\mathrm{CC}$ chemokines (containing two adjacent cysteine residues) attract both monocytes and lymphocytes and the receptors are designated CCR. Macrophage-tropic HIV strains (including most primary isolates from patients) use the CCR5 receptor, are therefore referred to as R5 tropic and are usually non-syncytium-inducing (NSI). To confuse matters further, there are also HIV strains (mostly patient isolates of primary $\mathrm{T}$ cell strains), which are able to use both CCR-5 and CXCR-4, referred to as R5X4 tropic. This classification should be considered as an approximation of reality. ${ }^{10,20}$

There is a fascinating clinical observation relating to all this molecular coreceptor wizardry. There are two known mutations of the CCR-5 gene in humans, both of which result in failure of the functional receptor to appear on the cell surface. People carrying two mutant copies of the CCR-5 gene have a protective advantage against HIV infection, and those with one copy of the mutant gene follow a long-term non-progressive disease course. $^{21,22}$ About $1-3 \%$ of European-descended populations are protected from HIV infection because they carry two mutant copies of the CCR-5 gene (CCR-5 del32) and $14 \%$ carry one copy. They do not suffer any obvious ill-effects from lacking this chemokine receptor. It is speculated that the mutation became fixed in the population during the fourteenth century, when it might have conferred some protection during the bubonic plague epidemic.

Knowing now how HIV gets into different cell types and that a mutant receptor for HIV is 
sufficient to prevent HIV infection gives researchers some hope for designing drugs that inhibit HIV entry into cells.

\section{HIV-induced immunosuppression}

Among the paradoxes of HIV research have been the facts that a) not enough virus is produced in the asymptomatic phase of the infection to cause the direct death of the vast number of $\mathrm{T}$ cells that are ultimately destroyed, and $\mathrm{b}$ ) immune dysfunction is apparent even before T cell counts have dropped significantly. It is now becoming clear that HIV's effects on the immune system are far more extensive than mere depletion of virally infected $\mathrm{T}$ cells; the disruption involves a variety of cell types, both infected and bystanders, plus cytokines and other soluble molecules.

Gp120 is the prime suspect in immune disruption, both on the viral surface and as soluble, extracellular protein (see Figure 2). When it binds to CD4 and chemokine receptors on $\mathrm{T}$ cells or antigen presenting cells, it can block binding of the normal ligands and therefore interferes with normal antigen presentation and normal chemokine signalling, and, it can lead to internalization of the receptors and therefore loss of surface receptors. Gp120, either free or when presented on the surface of antigen presenting cells, can also cause inappropriate activation of intracellular signalling pathways. This can lead to anergy or even

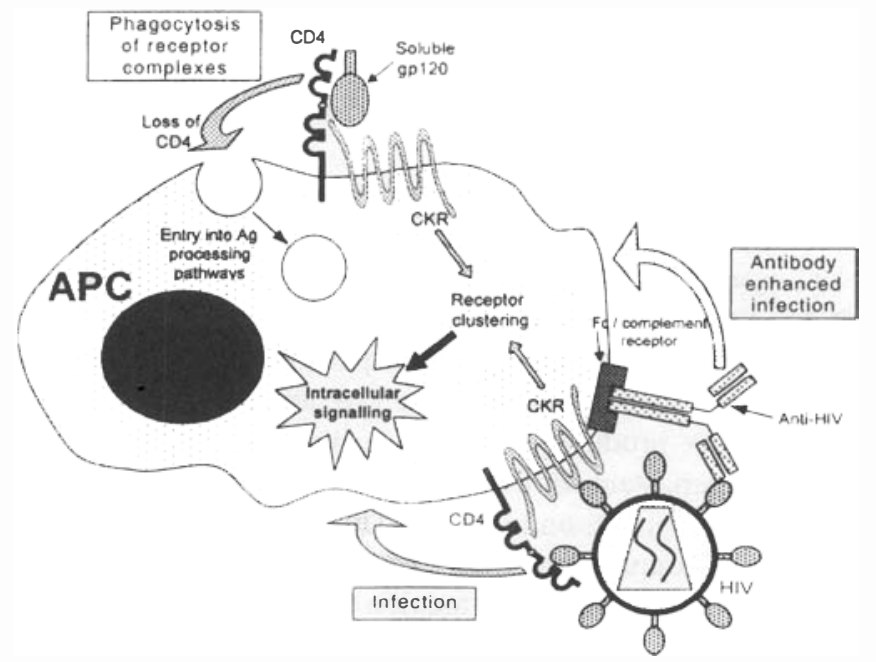

Figure 2. HIV-1 interactions with APC cell surface receptors. HIV-1 and its shed surface protein gp120 are able to interact with a number of receptor molecules on APC. The gp120 protein has binding sites for CD4 and chemokine receptors such as CCR5. It can also interact with Fc or complement receptors via anti-HIV-1 antibodies. Outcomes of HIV-1 proteins binding to APC receptors include infection of the cell by HIV-l (a process that can be enhanced by antibodies to HIV-l, antibody-enhanced infection), and phagocytosis of receptor complexes bound to soluble gp120, leading to the loss of receptors, such as CD4, and possible entry of the receptor/gp120 complex into antigen processing pathways. It should be noted that all of the HIV-1 receptors are linked to intracellular signalling pathways that can be activated on binding HIV-l or gp120 and that this can lead to disruption of the cell's function. CKR, chemokine receptor. [Reproduced with permission from Hewson T, Lone N, Moore M et al., Interactions of HIV-1 with antigen-presenting cells, Immunology and Cell Biology, 1999; 77: 289-303 (http://www.blackwell-science.com/icb).] 
activation-induced cell death (AICD, a form of apoptosis) being induced instead of cell stimulation, and is probably a major mechanism by which the functional depletion of $\mathrm{T}$ cells seen in HIV disease occurs. ${ }^{10,20,23}$ These kinds of antigen-specific mechanisms can explain how significant damage can be wreaked in the immune system even before the wholesale collapse in T cell numbers; crucial $\mathrm{T}$ cell clones that are needed to combat HIV and other concurrent infections are being selectively wiped out instead of being stimulated.

In addition to blatant $\mathrm{T}$ cell death and functional depletion, the immune system can also be unbalanced by changes in cytokine production in HIV infection. HIV infection of APCs modulates production of interferon, interleukin (IL)-10 and IL-12, which can induce a switch of $\mathrm{T}$ cell phenotypes from $\mathrm{T}$ helper (Th-1, associated with cell-mediated immune responses) to Th-2 (associated with humoral responses). This switch in dominant $\mathrm{T}$ cell subsets has been reported in patients as their disease progresses, but the hypothesis is probably rather simplistic. $^{10}$

The loss of $\mathrm{T}$ cell clones and the proposed shift in cytokine profile from Th- 1 to Th- 2 also compromises other, effector arms of the immune system. B cell dysregulation leads to spontaneous polyclonal proliferation and IgG secretion, but an impaired response to de novo antigens. ${ }^{24}$ Macrophage function, including phagocytosis and intracellular killing of bacteria, is impaired both by the change in cytokine profile and by the fact that macrophages themselves are infected by HIV. This is of particular relevance to mycobacteria, which live inside macrophages.

\section{Course of HIV infection}

Armed with the basic biological evidence described above, we can now suggest explanations for the course of HIV infection.

The (typically M-tropic) virus usually enters an individual by infecting macrophages, dendritic cells and other CD4+ cells in mucosal tissue. The virus is passed on to systemic activated $\mathrm{T}$ cells and a period of acute infection develops, where virus replication is high and can be easily detected in the blood and lymph nodes. An initial antiviral immune response then causes viral titres to drop drastically and a persistent state is established, which can continue for years. During this time there is a low level of virăl production (when new variants will be continually produced), kept in temporary check by the immune system. However, there is a slow, persistent decline in CD4+ lymphocytes. Eventually, more virulent viral strains (often T-tropic, syncytium inducing) emerge, which leads to a second high level of viraemia. This is usually coupled with a decline in T cell count to below 300 cells $/ \mu \mathrm{l}$, and is the point at which opportunistic infections manifest themselves. ${ }^{9}$

\section{Opportunistic infection in HIV-infected patients}

Viruses, fungi, bacteria and parasites all exploit the immunosuppressed environment of patients with AIDS, often leading to disseminated disease. The likelihood of opportunistic infection depends both upon the degree of immune dysfunction and on the environment in which the individual lives (and has lived, for those infections that are reactivated).

The most common viral infections in HIV patients are those of the herpes family. Such viruses can also enhance HIV production, not only by induction of cytokines, but also directly 
by transactivating factors within co-infected cells. Other viruses include ones associated with neoplasms, such as human papilloma virus. The B cell polyclonal activation induced by HIV is favourable for Epstein-Barr virus proliferation and can promote B-cell lymphomas. ${ }^{9}$

The likelihood of developing opportunistic fungal and parasitic infections in HIV depends largely on the geographical location of the patient and therefore their exposure to the pathogen. For example, exposure to Cryptococcus neoformans is universal (though particularly in Africa), whereas histoplasmosis occurs mainly in the United States of America. ${ }^{24}$ Toxoplasmosis and leishmaniasis are good examples of protozoal infections exacerbated or reactivated by HIV.

Bacteria thrive in the face of impaired antibody production and macrophage dysfunction induced by HIV. Poor mucosal defences encourage a range of enteric infections, such as Salmonella and Shigella, which can lead to bacteraemia. Since mycobacteria prefer to live inside macrophages, it is obvious that they will benefit from impaired macrophage function. M. avium, in particular, thrives and leads to disseminated disease, mostly when $\mathrm{T}$ cell counts are very low. It is perhaps surprising that $M$. leprae is not more of a problem in HIV. Slow multiplication rate of $M$. leprae means it cannot quickly colonize an immunocompromised host. There are only very few reports of some degree of association between HIV and leprosy ${ }^{7}$ and of HIV altering the clinical course of leprosy cases. ${ }^{5}$

Tuberculosis, on the other hand is remarkable because it can be reactivated somewhat earlier in HIV infection. This is either because it is a more virulent pathogen or because of involvement of more specific mechanisms, such as functional depletion of antigen-specific $\mathrm{T}$ cell clones which were holding latent infection in check. The proposed Th-1/Th- 2 switch would also facilitate the proliferation of mycobacteria (which are controlled more effectively by a Th1-mediated response). The question of whether there is a more specific relationship, on a cellular level, between mycobacteria and HIV infection, has been addressed by a few researchers. MAC replication is enhanced by HIV co-infection of cell cultures in vitro, although the mechanism behind this has not been elucidated. ${ }^{25-27}$ Conversely, lipoarabinomannan (LAM) from M.tb H37Ra (although less so for LAM from virulent M.tb strains) has been shown to stimulate HIV replication in macrophage cell lines both directly via NF- $\alpha \mathrm{B}$ and via TNF- $\alpha$ production. ${ }^{28,29}$ So we can see how HIV can facilitate mycobacterial survival, and that mechanisms exist whereby mycobacteria could facilitate HIV survival.

The terminal phase of HIV disease is therefore a vicious cycle of viral production, destruction of immune cells, repeated opportunistic infection and further immune dysfunction, from which the body cannot by itself escape.

\section{References}

1 UNAIDS, WHO. AIDS epidemic update: December 1998. World Health Organisation, Geneva, 1998.

2 Jacobson MA. Mycobacterium avium complex. In: Cohen PT, Sande MA, Volberding PA (eds) The AIDS knowledge base, 3rd edn. Lippincott Williams and Wilkins, Philadelphia, 1999.

3 Small PM, Scheter GF, Goodman PC et al. Treatment of tuberculosis in patients with advanced human immunodeficiency virus infection. N Engl J Med, 1991; 324: 289-294.

${ }^{4}$ Chambers HF. Mycobacterium tuberculosis infection. In: Cohen PT, Sande MA, Volberding PA (eds) The AIDS knowledge base, 3rd edn. Lippincott Williams and Wilkins, Philadelphia, 1999.

5 Arunthathi S, Ebenezer L, Kumuda C. Reversal reaction, nerve damage and steroid therapy in three multibacillary HIV positive petients. Lepr Rev, 1998; 69: 173-177.

${ }^{6}$ Flageul B. Leprosy and infection by the human immunodeficiency virus. Acta Leprol, 1997; 10: 129-130.

7 van den Broek J, Chum HJ, Swai R et al. Association between leprosy and HIV infection in Tanzania. Int J Lepr Other Mycobact Dis, 1997; 65: 203-210. 
${ }^{8}$ Andrade VL, Moreira-Alves T, Regazzi-Avelleira JC et al. Prevalence of HIV1 in leprosy patients in Rio de Janeiro, Brazil. Acta Leprol, 1997; 10: 159-163.

9 Levy JA. HIV and the pathogenesis of AIDS, 2nd edn. ASM Press, Washington DC, 1998.

${ }^{10}$ Hewson T, Lone N, Moore M et al. Interactions of HIV-1 with antigen-presenting cells. Immunol Cell Biol, 1999; 77: 289-303.

11 Dalgleish AG, Beverley PCL, Clapham PR et al. The CD4 (T4) antigen is an essential component of the receptor for the AIDS retrovirus. Nature, 1984; 312: 763-767.

12 Kwong PD, WYatt R, Robinson J et al. Structure of an HIV gp120 envelope glycoprotein in complex with the CD4 receptor and a human neutralising antibody. Nature, 1998; 393: 648-659.

13 Cocchi F, DeVico AL, Garzino-Demo A et al. Identification of RANTES, MIP-1 alpha and MIP-1 beta as the major HIV-suppressive factors produced by CD8+ T cells. Science, 1995; 270: 1811-1815.

14 Alkhatib G, Combadiere C, Broder CC et al. CC CKR5: a RANTES, MIP-1 alpha, MIP-1 beta receptor as a fusion cofactor for macrophage-tropic HIV-1. Science, 1996; 272: 1955-1958.

15 Deng H, Liu R, Ellmeier W et al. Identification of a major co-receptor for primary isolates of HIV-1. Nature, 1996; 381: $661-666$.

16 Drajic T, Litwin V, Allaway GP et al. HIV-1 entry into CD4+ cells is mediated by the chemokine receptor CCCKR-5. Nature 1996; 381: 667-673.

17 Feng Y, Broder C, Kennedy PE et al. HIV-1 entry cofactor: functional cDNA cloning of a seven-transmembrane, G protein-coupled receptor. Science, 1996; 272: 872-877.

18 De Jong JJ, De Ronde A, Keulen W et al. Minimal requirements for the human immunodeficiency virus type 1 V3 domain to support the syncytium-inducing phenotype: analysis by single amino acid substitution. $J$ Virol, 1992 ; 66: $6777-6780$.

19 Isaka Y, Sato A, Miki S et al. Small amino acid changes in the V3 loop of human imunodeficiency virus type 2 determines the coreceptor usage for CXCR4 and CCR5. Virology, 1999; 264: 237-243.

${ }^{20}$ Norcross MA. Chemokine receptors and HIV-1 pathogenesis: a viral fatal attracion. In: Dalgliesh A, Weiss R (eds) HIV and the new viruses, 2nd edn. Academic Press, New York, 1999.

${ }^{21}$ Michael NL, Chang G, Louie LG et al. The role of viral phenotype and CCR-5 gene defects in HIV-1 transmission and disease progression. Nat Med, 1997; 3: 338-340.

22 Michael NL. Host genetic influences on HIV-1 pathogenesis. Curr Opin Immunol, 1999; 11: 466-474.

${ }^{23}$ Cottrez F, Manca F, Dalgleish AG et al. Priming of human CD4(+) antigen-specific T cells to undergo apoptosis by HIV-infected monocytes - a two-step mechanism involving the gp120 molecule. J Clin Invest, 1997; 99: 257-266.

${ }^{24} \mathrm{Neu}$ HC, Ortiz-Neu C. Opportunistic infections in HIV-infected patients. In: Neu HC, Levy JA and Weiss RA (eds) Focus on HIV. Churchill Livingstone, London, 1993.

25 Kallenius G, Kovula T, Rydgard KJ et al. Human immunodeficiency virus type 1 enhances intracellular growth of Mycobacterium avium in human macrophages. Infect Immun, 1992; 60: 2453-2457.

${ }^{26}$ Newman GW, Kelley TG, Gan H et al. Concurrent infection of human macrophages with HIV-1 and Mycobacterium avium results in decreased cell viability, increased $M$. avium multiplication and altered cytokine production. J Immunol, 1993; 151: 2261-2272.

27 Ghassemi M, Andersen BR, Reddy VM et al. Human Immunodeficiency virus and Mycobacterium avium complex coinfection of monocytoid cells results in reciprocal enhancement of multiplication. J Infect Dis, 1995; 171: $68-73$.

${ }^{28}$ Zhang Y, Nakata K, Weiden M et al. Mycobacterium tuberculosis enhances human immunodeficiency virus-1 replication by transcriptional activation at the long terminal repeat. J Clin Invest, 1995; 95: 2324-2331.

29 Brown MC, Taffet SM. Lipoarabinomannans derived from different strains of Mycobacterium tuberculosis differentially stimulate the activation of NF- $\alpha \mathrm{B}$ and KBF1 in murine macrophages. Infect Immun, 1995; 63: 1960-1968. 